CIRJE-F-292

\title{
Some Improvements in Numerical Evaluation of Symmetric Stable Density and its Derivatives
}

Muneya Matsui

Akimichi Takemura

The University of Tokyo

August 2004

CIRJE Discussion Papers can be downloaded without charge from:

http://www.e.u-tokyo.ac.jp/cirje/research/03research02dp.html

Discussion Papers are a series of manuscripts in their draft form. They are not intended for circulation or distribution except as indicated by the author. For that reason Discussion Papers may not be reproduced or distributed without the written consent of the author. 


\title{
Some improvements in numerical evaluation of symmetric stable density and its derivatives
}

\author{
Muneya MATSUI ${ }^{\dagger}$ and Akimichi TAKEMURA ${ }^{\ddagger}$ \\ $\dagger$ Graduate School of Economics, University of Tokyo \\ $\ddagger$ Graduate School of Information Science and Technology, University of Tokyo
}

\begin{abstract}
We propose improvements in numerical evaluation of symmetric stable density and its partial derivatives with respect to the parameters. They are useful for more reliable evaluation of maximum likelihood estimator and its standard error. Numerical values of the Fisher information matrix of symmetric stable distributions are also given. Our improvements consist of modification of the method of Nolan (1997) for the boundary cases, i.e., in the tail and mode of the densities and in the neighborhood of the Cauchy and the normal distributions.
\end{abstract}

\section{Introduction}

There have been many researches to evaluate densities and quantiles of symmetric or general stable distributions. McCulloch (1998) considered efficient algorithms for approximating symmetric stable densities $f(x ; \alpha)$ for the range $\alpha>0.85$, where parameter $\alpha$ denotes the characteristic exponent. Nolan (1997) gave accurate algorithms for general stable densities based on integral representations of the densities which were derived by Zolotarev (1986). Nolan provides a very useful program package "STABLE" on his web page*. However his program exhibits some unreliable behavior around the boundary as stated in the users guide of STABLE. Therefore even in the case of symmetric stable distributions, reliable computations of density functions including all the boundary cases is still needed. Furthermore for maximum likelihood estimation, it is desirable to directly compute the derivatives of the density function. In this paper we present reliable computations of symmetric stable density functions and their partial derivatives. Our computation of densities is accurate for all values of $x$ and $0.1<\alpha \leq 2$. Concerning the partial derivatives it is accurate in a somewhat smaller range of values.

Regarding maximum likelihood estimation for the range $\alpha \geq 0.4$, Nolan (2001) used interpolated stable densities and maximized the likelihood by approximate gradient search (constrained quasiNewton method) because of its efficiency. But near the boundary of the parameter space interpolation may be inaccurate and the direct integral representation is used. Note that the direct integral representation is also not very reliable and slow near the boundary. In the symmetric case, Brorsen and Yang (1990) discussed maximum likelihood estimation using an integral representation of the densities given by Zolotarev (1986). But they have only considered the range $\alpha>1$ to avoid the discontinuity and nondifferentiability at $\alpha=1$. Furthermore they did not check the sample covariances of their maximum likelihood computation with the Fisher information matrix. These previous researches on

\footnotetext{
${ }^{*}$ http://academic2 . american.edu/ ${ }^{\sim}$ jpnolan
} 
maximum likelihood estimation have not used direct evaluation of the derivatives of the log likelihood function with respect to the parameters. For reliable evaluation of the maximum likelihood estimator and its standard error, direct and reliable evaluation of the first and the second derivatives of the log likelihood function is desirable.

The organization of this paper is as follows. In Section 2 we summarize notations and preliminary results on symmetric stable density. In Section 3 we provide an accurate algorithm for calculations of symmetric stable distributions which modifies Nolan (1997) for $x$ near 0 or $\infty$ and for $\alpha=1$ or $\alpha=2$ using various expansions. Accurate algorithms for the partial derivatives of symmetric stable distributions with respect to the parameters are given in Section 4 and Section 5. Fisher information matrices are calculated in Section 6, together with some simulation studies on the variance of the maximum likelihood estimator and the observed Fisher information. We also discuss behavior of Fisher information as $\alpha \rightarrow 2$. Some discussions are given in Section 7 .

\section{Preliminaries}

In this section we prepare notations and summarize preliminary results. There are many parameterizations for stable distributions and much confusion has been caused. Our parameterizations follow the useful parameterizations for statistical inference which were given in Nolan (1998).

\section{$2.1 \quad$ Notations}

Let

$$
\Phi(t)=\Phi(t ; \mu, \sigma, \alpha)=\exp \left(-|\sigma t|^{\alpha}+i \mu t\right)
$$

denote the characteristic function of symmetric stable distribution with parameters

$$
\theta=\left(\theta_{1}, \theta_{2}, \theta_{3}\right)=(\mu, \sigma, \alpha)
$$

where $\alpha$ is the characteristic exponent, $\mu$ is a location parameter and $\sigma$ is a scale parameter. For the standard case $(\mu, \sigma)=(0,1)$ we simply write the characteristic function as $\Phi(t ; \alpha)=\exp \left(-|t|^{\alpha}\right)$.

The corresponding density is written as $f(x ; \mu, \sigma, \alpha)$ and $f(x ; \alpha)$ in the standard case:

$$
f(x ; \mu, \sigma, \alpha)=\frac{1}{\sigma} f\left(\frac{x-\mu}{\sigma} ; \alpha\right) .
$$

At $\alpha=1$

$$
f(x ; 1)=\frac{1}{\pi\left(1+x^{2}\right)}
$$

is the Cauchy density and at $\alpha=2$

$$
f(x ; 2)=\frac{1}{2 \sqrt{\pi}} \exp \left(-x^{2} / 4\right)
$$

is the normal density $N(0,2)$. Accordingly the density can be defined to constitute a location-scale family. In the following, the first derivative of $f(x ; \alpha)$ with respect to $x$ is denoted by $f^{\prime}(x ; \alpha)$ and the second derivative is denoted by $f^{\prime \prime}(x ; \alpha)$. Then

$$
\begin{aligned}
& \frac{\partial}{\partial x} f(x ; \mu, \sigma, \alpha)=-\frac{\partial}{\partial \mu} f(x ; \mu, \sigma, \alpha)=\frac{1}{\sigma^{2}} f^{\prime}\left(\frac{x-\mu}{\sigma} ; \alpha\right), \\
& \frac{\partial^{2}}{\partial x^{2}} f(x ; \mu, \sigma, \alpha)=\frac{\partial^{2}}{\partial \mu^{2}} f(x ; \mu, \sigma, \alpha)=\frac{1}{\sigma^{3}} f^{\prime \prime}\left(\frac{x-\mu}{\sigma} ; \alpha\right) .
\end{aligned}
$$


The partial derivatives with respect to $\sigma$ and $\alpha$ are written by subscripts, e.g.,

$$
f_{\alpha}(x ; \mu, \sigma, \alpha)=\frac{\partial}{\partial \alpha} f(x ; \mu, \sigma, \alpha), \quad f_{\alpha \sigma}(x ; \mu, \sigma, \alpha)=\frac{\partial^{2}}{\partial \alpha \partial \sigma} f(x ; \mu, \sigma, \alpha) .
$$

As above, when these derivatives are evaluated at the standard case $(\mu, \sigma)=(0,1)$ we write $f_{\alpha}(x ; \alpha)$, $f_{\alpha \sigma}(x ; \alpha)$, etc. Note that

$$
f_{\sigma}(x ; \alpha)=-f(x ; \alpha)-x f^{\prime}(x ; \alpha), \quad f_{\sigma \sigma}(x ; \alpha)=2 f(x ; \alpha)+4 x f^{\prime}(x ; \alpha)+x^{2} f^{\prime \prime}(x ; \alpha) .
$$

Furthermore we write

$$
f_{\alpha}^{\prime}(x ; \alpha)=\frac{\partial^{2}}{\partial x \partial \alpha} f(x ; \alpha) .
$$

The reason we consider up to the second order derivatives of the density function is that in assessing the standard error of the maximum likelihood estimator $\hat{\theta}$, the observed Fisher information

$$
\hat{I}_{\theta \theta}\left(x_{1}, \ldots, x_{n}\right)=-\frac{1}{n} \sum_{i=1}^{n} \frac{\partial^{2} \log f\left(x_{i} ; \hat{\theta}\right)}{\partial \theta^{2}}=\frac{1}{n} \sum_{i=1}^{n}\left(\frac{\frac{\partial}{\partial \theta} f\left(x_{i} ; \hat{\theta}\right)}{f\left(x_{i} ; \hat{\theta}\right)}\right)^{2}-\frac{1}{n} \sum_{i=1}^{n} \frac{\frac{\partial^{2}}{\partial \theta^{2}} f\left(x_{i} ; \hat{\theta}\right)}{f\left(x_{i} ; \hat{\theta}\right)}
$$

is usually preferred to the value of the Fisher information matrix at $\hat{\theta}$ (e.g. Efron and Hinkley (1978)).

Note that there are other parameterizations of symmetric stable distributions than (2.1). However different parameterizations in the literature are smooth functions of each other including the boundary $\alpha=2$ and differentiations in terms of other parameterizations can be obtained from the results of this paper by the chain rule of differentiation.

\section{$2.2 \quad$ Preliminary results}

From equation (2.2.18) of Zolotarev (1986) or Theorem 1 of Nolan (1997), the density $f(x ; \alpha)$ for the case $\alpha \neq 1$ and $x>0$ is written as

$$
f(x ; \alpha)=\frac{\alpha}{\pi|\alpha-1| x} \int_{0}^{\frac{\pi}{2}} g(\varphi ; \alpha, x) \exp (-g(\varphi ; \alpha, x)) d \varphi,
$$

where

$$
g(\varphi ; \alpha, x)=\left(\frac{x \cos \varphi}{\sin \alpha \varphi}\right)^{\frac{\alpha}{\alpha-1}} \frac{\cos (\alpha-1) \varphi}{\cos \varphi}
$$

Note that at $x=0$

$$
f(0 ; \alpha)=\frac{1}{\pi} \Gamma\left(1+\frac{1}{\alpha}\right)
$$

for all $0<\alpha \leq 2$.

For the case $x \rightarrow 0$ and $0<\alpha \leq 2, \alpha \neq 1$, the following expansion can be used.

$$
f(x ; \alpha)=\frac{1}{\pi} \sum_{k=0}^{\infty} \frac{\Gamma((2 k+1) / \alpha+1)}{(2 k+1) !}(-1)^{k} x^{2 k}=\frac{1}{\pi \alpha} \sum_{k=0}^{\infty} \frac{\Gamma((2 k+1) / \alpha)}{(2 k) !}(-1)^{k} x^{2 k} .
$$


For $\alpha<1$, this series is not convergent but can be justified as an asymptotic expansion as $x \rightarrow 0$. For $1<\alpha \leq 2$, it is convergent for every $x$. Similarly for the case $x \rightarrow \infty$ and $0<\alpha \leq 2, \alpha \neq 1$, we have

$$
f(x ; \alpha)=\frac{1}{\pi} \sum_{k=1}^{\infty} \frac{\Gamma(k \alpha+1)}{k !}(-1)^{k-1} \sin \left(\frac{\pi \alpha k}{2}\right) x^{-k \alpha-1} .
$$

For $\alpha<1$ this series converges for every $x \neq 0$ and for $\alpha>1$ this series can be justified as an asymptotic expansion as $x \rightarrow \infty$. For $\alpha=2$ this asymptotic expansion is zero, which corresponds to the fact that the tail of normal distribution is exponentially small. These (asymptotic) expansions are stated in Bergström (1953), Section XVII.6 of Feller (1971), Section 2.4 and Section 2.5 of Zolotarev (1986).

\section{Numerical evaluation of symmetric stable densities}

As in Nolan (1997) we numerically evaluate the density function using (2.6). In (2.6) the function $g(\varphi ; \alpha, x):\left[0, \frac{\pi}{2}\right] \rightarrow[0, \infty]$ plays an important role, because properties of this function make the numerical integration quite efficient. Note that $g(\varphi ; \alpha, x)$ is continuous and positive, strictly increases from 0 to $\infty$ for $\alpha<1$ and strictly decreases from $\infty$ to 0 for $\alpha>1$. Therefore the integrand $g(\cdot) \exp (-g(\cdot))$ is unimodal and its maximum value $1 / e$ is uniquely attained at $\varphi_{1}$ satisfying $g\left(\varphi_{1} ; \alpha, x\right)=1$. When the value of density is small, the integrand concentrates around its mode very narrowly. Then quadrature algorithms may miss the integrand. Therefore we solve $g(\varphi ; \alpha, x)=1$ for $\varphi_{1}$ and the integral is divided into two intervals around this mode (see Nolan (1997)). For numerical calculations of (2.6) we use adaptive integration with singularity (QAGS) in GNU Scientific Library (2003). For most values of $x$ and $\alpha$ this integration works well.

However, when $\alpha$ is close to 2 this algorithm has some difficulty. Note that for $\alpha=2$

$$
g(\varphi ; 2, x)=\left(\frac{x}{2 \sin \varphi}\right)^{2}
$$

and $g(\pi / 2 ; 2, x)=x / 2$. Therefore $\varphi_{1}$ exceed $\pi / 2$ when $x>2$. There are some other numerical difficulties in (2.6). We list these difficulties and propose alternative practical methods for evaluating the density.

1. $\alpha$ is small and $x \rightarrow 0$ :

If $\alpha$ is small, the density is very much concentrated at $x=0$. For example Nolan (1997) states $f(0 ; 0.1)=1.155 \times 10^{6}$ whereas $f(0.01 ; 0.1)=1.66$. In our calculations when $\alpha$ is small and $x$ near 0 the values of $(2.6)$ sometimes become larger than $f(0 ; \alpha)$, contradicting the unimodality of the stable density. For this case we can use the asymptotic expansion (2.8).

2. $x \rightarrow \infty$ :

We cannot guarantee the accuracy of (2.6) in the case of $x \rightarrow \infty$. Since stable distributions have heavy tails, reliable calculation of their densities is needed for large $x$. In our calculations when $\alpha>1$ and $x$ is large the values of (2.6) sometimes become much smaller than the asymptotic expansion (2.9). For this case we can use the asymptotic expansion (2.9).

3. $\alpha$ is near 1 :

The representation (2.6) can not be applied at $\alpha=1$ theoretically. The numerical quadrature of (2.6) becomes unreliable because of roundoff errors, when $\alpha$ is close to 1 . 
4. $\alpha$ is near 2 :

Though the representation (2.6) can be applied near $\alpha=2$ theoretically, it seems to be too close to the normal distribution in the tail of the distribution. Actually the values of the density in the tail obtained by the integral representation (2.6) is much smaller than the asymptotic expansion (2.9).

For the rest of this section, we discuss the cases 3 and 4 above.

For $\alpha \doteq 1$, we consider Taylor expansion of the density around $\alpha=1$. Let

$$
\gamma \doteq 0.57722
$$

denote the Euler's constant throughout the rest of this paper. The Taylor expansion of $f(x ; \alpha)$ around $\alpha=1$ is given as follows.

$$
f(x ; \alpha)=f(x ; 1)+f_{\alpha}(x ; 1)(\alpha-1)+\frac{1}{2} f_{\alpha \alpha}(x ; 1)(\alpha-1)^{2}+\frac{1}{6} f_{\alpha \alpha \alpha}(x ; 1)(\alpha-1)^{3}+o\left((\alpha-1)^{3}\right),
$$

where

$$
\begin{aligned}
f_{\alpha}(x ; 1)= & \frac{1}{\pi}\left\{\frac{x^{2}-1}{\left(1+x^{2}\right)^{2}}\left(1-\gamma-\frac{1}{2} \log \left(1+x^{2}\right)\right)+\frac{2 x}{\left(1+x^{2}\right)^{2}} \arctan x\right\} \\
f_{\alpha \alpha}(x ; 1)= & \frac{x^{4}-6 x^{2}+1}{\pi\left(1+x^{2}\right)^{3}}\left\{\frac{\pi^{2}}{6}+\left(1-\gamma-\frac{1}{2} \log \left(1+x^{2}\right)\right)^{2}-1-\arctan ^{2} x\right\} \\
& +\frac{8 x\left(x^{2}-1\right)}{\pi\left(1+x^{2}\right)^{3}} \arctan x\left(\frac{3}{2}-\gamma-\frac{1}{2} \log \left(1+x^{2}\right)\right) \\
& +\frac{2}{\pi\left(1+x^{2}\right)^{3}}\left\{\left(1-3 x^{2}\right)\left(1-\gamma-\frac{1}{2} \log \left(1+x^{2}\right)\right)-x\left(1+x^{2}\right) \arctan x\right\}
\end{aligned}
$$

For convenience the explicit form of $f_{\alpha \alpha \alpha}(x ; 1)$ is given in appendix A. (3.2) and (3.3) are proved as follows. From the equation 4.40 on page 18 of Oberhettinger (1990)

$$
\int_{0}^{\infty} u^{\nu-1} e^{-a u} \log u \cos (u y) d u=\left(a^{2}+y^{2}\right)^{-\frac{1}{2} \nu} \Gamma(\nu)\left[\cos (\nu z)\left\{\psi(\nu)-\frac{1}{2} \log \left(a^{2}+y^{2}\right)\right\}-z \sin (\nu z)\right]
$$

where

$$
\operatorname{Re} \nu>0, \quad z=\arctan (y / a), \quad \psi(\nu)=\Gamma^{\prime}(\nu) / \Gamma(\nu)
$$

Differentiating (3.4) several times with respect to $\nu$, setting $\nu=2$, and combining the results in the inversion formula we obtain (3.2) and (3.3). The conditions for change of integral and differentiation are satisfied in these cases. Although higher order derivatives of $f(x ; \alpha)$ with respect to $\alpha$ can be evaluated along the same line, we found that the three term expansion (3.1) is sufficiently accurate.

Now we consider the case $\alpha \rightarrow 2$ and $x \rightarrow \infty$. It seems natural to use asymptotic expansion (2.9). However the normal density has an exponentially small tail and this expansion is meaningless for $\alpha=2$. However in view of smoothness at $\alpha=2$, an approximation around the normal density is desirable. This case is somewhat subtle, but we found that the following procedure works well numerically. Note that from $(2.8)$, for each fixed $x, f(x ; \alpha)$ is differentiable with respect to $\alpha(>1)$ even at $\alpha=2$, i.e., for each fixed $x$ we have

$$
f(x ; \alpha)=f(x ; 2)+f_{\alpha}(x ; \alpha)(\alpha-2)+\frac{1}{2} f_{\alpha \alpha}(x ; \alpha)(\alpha-2)^{2}+o\left((\alpha-2)^{2}\right) .
$$


Differentiating (2.9) with respect to $\alpha$, for large $x$, heuristically we have

$$
f_{\alpha}(x ; \alpha)(\alpha-2) \sim \frac{1}{\pi} \Gamma(\alpha+1) \frac{\pi}{2} \cos \left(\frac{\pi \alpha}{2}\right) x^{-\alpha-1}(\alpha-2) \sim \frac{\Gamma(\alpha+1)}{2} x^{-\alpha-1}(2-\alpha)
$$

and

$$
f(x ; \alpha) \sim f_{\alpha}(x ; \alpha)(\alpha-2) .
$$

We summarize our treatments of various boundary cases in Table 1 . The range of $x$ and $\alpha$ and the number of terms $k$ in the expansions (2.8) and (2.9) are shown. For $0.99<\alpha \leq 1.01$ we use formula (3.1) and for $\alpha>1.99999$ and for $x$ large we use the maximum of (2.6) and (3.5). Note that for most cases a small number of terms in the expansions (2.8) or (2.9) is sufficient. The approximations are very effective since the expansions and the integral (2.6) give virtually the same results for most values of $x$ and $\alpha$.

\begin{tabular}{|c|c|c|c|c|}
\hline$\alpha \backslash x$ & \multicolumn{2}{|c|}{$x \rightarrow 0$, formula $(2.8)$} & \multicolumn{2}{|c|}{$x \rightarrow \infty$, formula $(2.9)$} \\
\hline & number of terms $k$ & range of $x$ & number of terms $k$ & range of $x$ \\
\hline$[0.1,0.2]$ & $k=1$ & $x<10^{-16}$ & \multirow{3}{*}{$k=10$} & \multirow{3}{*}{$x>10^{\frac{3}{1+\alpha}}$} \\
\hline$(0.2,0.5]$ & \multirow{2}{*}{$k=5$} & $x<10^{-8}$ & & \\
\hline$(0.5,0.99]$ & & $x<10^{-5}$ & & \\
\hline$(0.99,1.01]$ & \multicolumn{4}{|c|}{ formula (3.1) } \\
\hline$(1.01,1.99999]$ & $k=10$ & $x<10^{-5}$ & $k=10$ & $x>10^{\frac{3}{1+\alpha}}$ \\
\hline$(1.99999,2.0]$ & $k=85$ & $x \leq 7$ & \multicolumn{2}{|c|}{$\max ((2.6),(3.5))$} \\
\hline
\end{tabular}

Table 1: Approximations to stable density at boundary cases

\section{Partial derivatives of the symmetric stable density with respect to the location and the scale parameters}

In this section we discuss the first and the second derivatives of the stable density with respect to the location parameter $\mu$ and the scale parameter $\sigma$.

\subsection{The first derivatives w.r.t. location and scale}

Differentiating (2.6) we obtain

$$
f^{\prime}(x ; \alpha)=-\frac{1}{x} f(x ; \alpha)+\frac{\alpha^{2} \operatorname{sign}(\alpha-1)}{\pi x^{2}(\alpha-1)^{2}} \int_{0}^{\frac{\pi}{2}} g(\varphi ; \alpha, x)(1-g(\varphi ; \alpha, x)) \exp (-g(\varphi ; \alpha, x)) d \varphi
$$

for $\alpha \neq 1$ and $x>0$. In view of (2.2) and (2.4) we only have to evaluate (4.1). The integrand $g(\cdot)(1-g(\cdot)) \exp (-g(\cdot))$ has a positive local maximum and a negative local minimum. These are attained at $\varphi_{2}: g\left(x ; \alpha, \varphi_{2}\right)=\frac{3-\sqrt{5}}{2}$ (local maximum) and $\varphi_{3}: g\left(x ; \alpha, \varphi_{3}\right)=\frac{3+\sqrt{5}}{2}$ (local minimum). For $\alpha<1$ the ordering of these points is $\varphi_{3} \leq \varphi_{1} \leq \varphi_{2}$ and the reverse holds for $\alpha>1$. Accordingly we divide the interval of integration and then the method of adaptive integration gives reliable values. Without these divisions the numerical integration sometimes does not converge or produces incorrect 
values. Note that if $\alpha$ is very close to 2 , the values of $\varphi_{i}, i=1,2,3$, are outside of the integration range. Therefore another treatment is needed for this case.

As in the case of the density itself, we use alternative representations of the first derivative of the symmetric stable density around the boundary and following representations are needed.

1. Expansions of the first derivative of the density.

$$
f^{\prime}(x ; \alpha)=\frac{1}{\pi \alpha} \sum_{k=1}^{\infty} \frac{\Gamma((2 k+1) / \alpha)}{(2 k-1) !}(-1)^{k} x^{2 k-1} .
$$

As in (2.8), for $1<\alpha \leq 2$, this series is convergent for every $x$. For $\alpha<1$, this series is justified as an asymptotic expansion as $x \rightarrow 0$. Similarly for the case $x \rightarrow \infty$ we have

$$
f^{\prime}(x ; \alpha)=\frac{1}{\pi} \sum_{k=1}^{\infty} \frac{\Gamma(\alpha k+2)}{k !}(-1)^{k} \sin \left(\frac{\pi \alpha k}{2}\right) x^{-k \alpha-2} .
$$

For $\alpha<1$ this series converges for every $x \neq 0$ and for $\alpha>1$ this series is justified as an asymptotic expansion.

2. Taylor expansion of derivative of density around $\alpha=1$ :

$$
f^{\prime}(x ; \alpha)=f^{\prime}(x ; 1)+f_{\alpha}^{\prime}(x ; 1)(\alpha-1)+\frac{1}{2} f_{\alpha \alpha}^{\prime}(x ; 1)(\alpha-1)^{2}+o\left((\alpha-1)^{2}\right),
$$

where

$$
\begin{gathered}
f^{\prime}(x ; 1)=-\frac{2 x}{\pi\left(1+x^{2}\right)^{2}}, \\
f_{\alpha}^{\prime}(x ; 1)=\frac{1}{\pi}\left\{\frac{-2 x^{3}+6 x}{\left(1+x^{2}\right)^{3}}\left(\frac{3}{2}-\gamma-\frac{1}{2} \log \left(1+x^{2}\right)\right)+\frac{2-6 x^{2}}{\left(1+x^{2}\right)^{3}} \arctan x\right\},
\end{gathered}
$$

and

$$
\begin{aligned}
f_{\alpha \alpha}^{\prime}(x ; 1)= & -\frac{2 x\left(x^{4}-14 x^{2}+9\right)}{\pi\left(1+x^{2}\right)^{4}}\left\{\frac{\pi^{2}}{6}+\left(1-\gamma-\frac{1}{2} \log \left(1+x^{2}\right)\right)^{2}-1-\arctan ^{2} x\right\} \\
& -\frac{8\left(3 x^{4}-8 x^{2}+1\right)}{\pi\left(1+x^{2}\right)^{4}} \arctan x\left(\frac{3}{2}-\gamma-\frac{1}{2} \log \left(1+x^{2}\right)\right) \\
& -\frac{2 x\left(x^{4}-22 x^{2}+17\right)}{\pi\left(1+x^{2}\right)^{4}}\left(1-\gamma-\frac{1}{2} \log \left(1+x^{2}\right)\right) \\
& -\frac{4\left(x^{4}-6 x^{2}+1\right)}{\left(1+x^{2}\right)^{4}} \arctan x+\frac{8 x\left(x^{2}-1\right)}{\left(1+x^{2}\right)^{4}} .
\end{aligned}
$$

Table 2 is a summary of approximations to the first derivative $f^{\prime}(x ; \alpha)$. The interpretation of Table 2 is almost the same as Table 1. Note that for $0.99<\alpha \leq 1.01$ the approximation (4.4) around Cauchy using Taylor expansion to the order $O\left((\alpha-1)^{2}\right)$ is accurate enough. In the range $\alpha \in[0.1,0.2)$, approximations are not good and we do not consider accurate calculations of derivatives. For $\alpha$ very close to 2 and $x$ large, we have the same problem as in the case of the density. However (4.1) gives reasonable values and we use (4.1). Note that if $\alpha$ is very close to 2 , then we do not observer very 
Table 2: Approximations to $f^{\prime}(x ; \alpha)$ at boundary cases

\begin{tabular}{|c|c|c|c|c|}
\hline$\alpha \backslash x$ & $x \rightarrow 0$, formu & $(4.2)$ & $x \rightarrow \infty$, formu & $\mathrm{a}(4.3)$ \\
\hline & number of terms $k$ & range of $x$ & number of terms $k$ & range of $x$ \\
\hline$[0.2,0.25]$ & \multirow{3}{*}{$k=5$} & $x<10^{-8}$ & \multirow{3}{*}{$k=10$} & \multirow{3}{*}{$x>10^{\frac{3}{1+\alpha}}$} \\
\hline$(0.25,0.3]$ & & $x<10^{-6}$ & & \\
\hline$(0.3,0.99]$ & & $x<10^{-5}$ & & \\
\hline$(0.99,1.01]$ & \multicolumn{4}{|c|}{ formula (4.4) } \\
\hline$(1.01,1.99999]$ & \multirow{2}{*}{$k=10$} & \multirow{2}{*}{$x<10^{-3}$} & $k=10$ & $x>10^{\frac{3}{1+\alpha}}$ \\
\hline$(1.99999,2.0]$ & & & \multicolumn{2}{|c|}{ formula (4.1) } \\
\hline
\end{tabular}

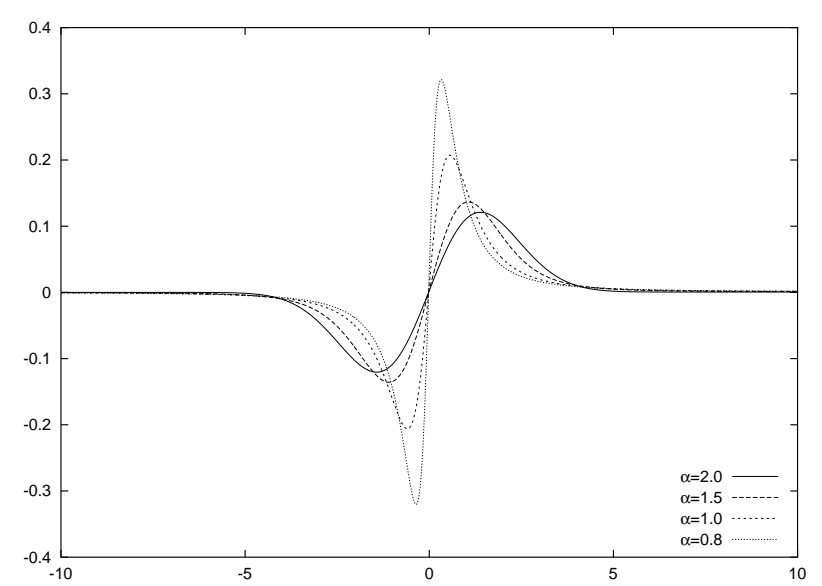

Figure 1: Derivative of density w.r.t. $\mu$

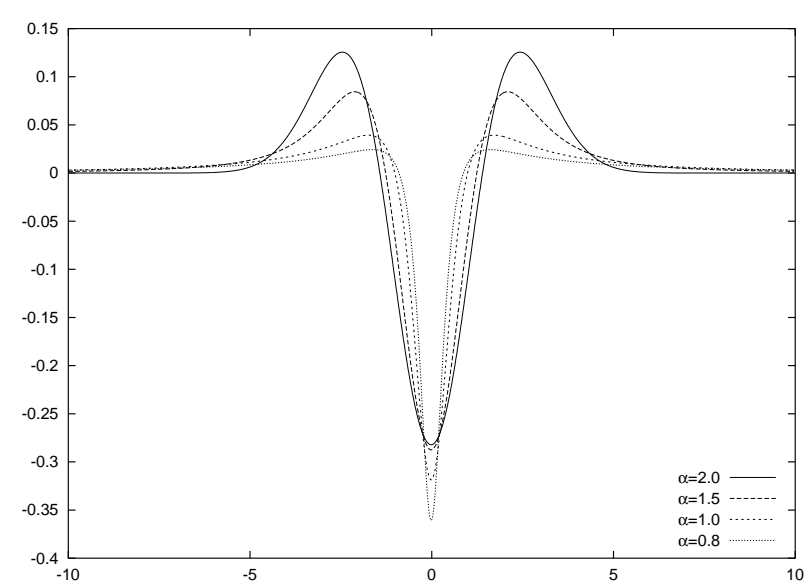

Figure 2: Derivative of density w.r.t. $\sigma$ 
large $x$ and the approximation to $f^{\prime}(x ; \alpha)$ is less important than the density itself. We will discuss this problem again in Section 6 in connection with accurate evaluation of the Fisher information for $\alpha$ very close to 2 .

We present the graphs of the derivative concerning $\mu$ (Figure 1), and the derivative concerning $\sigma$ (Figure 2). Both derivatives are continuous in $\alpha$ and we do not see abrupt changes of the forms for different values of $\alpha$.

\subsection{The second derivatives w.r.t. location and scale}

We only need to evaluate $f^{\prime \prime}(x ; \alpha)$ as in the case of the first derivatives. Differentiating (4.1) we obtain

$$
\begin{aligned}
f^{\prime \prime}(x ; \alpha)= & \frac{1}{\alpha-1} \frac{1}{x^{2}} f(x ; \alpha)+\frac{3-2 \alpha}{\alpha-1} \frac{1}{x} f^{\prime}(x ; \alpha) \\
& -\frac{\alpha^{3} \operatorname{sign}(\alpha-1)}{\pi(\alpha-1)^{3} x^{3}} \int_{0}^{\frac{\pi}{2}} g^{2}(\varphi ; \alpha, x)(2-g(\varphi ; \alpha, x)) \exp (-g(\varphi ; \alpha, x)) d \varphi .
\end{aligned}
$$

Considering that $f(x ; \alpha)$ and $f^{\prime}(x ; \alpha)$ can be calculated as in the previous sections, we only need to evaluate the integral which appear in the third term of $(4.5)$. The integrand $g^{2}(\cdot)(2-g(\cdot)) \exp (-g(\cdot))$ in the second term on the right hand side of (4.5) has a zero at $\varphi_{4}: g\left(x ; \alpha, \varphi_{4}\right)=2$, a local minimum at $\varphi_{1}: g\left(x ; \alpha, \varphi_{1}\right)=1$ and a local maximum at $\varphi_{5}: g\left(x ; \alpha, \varphi_{5}\right)=4$. For $\alpha<1$ the order of these points is $\varphi_{1} \leq \varphi_{4} \leq \varphi_{5}$ and the reverse order holds for $\alpha>1$. Therefore we can divide the interval of integral according to these values and get accurate results. Alternative representations of the second derivative of the symmetric stable density around the boundary are as follows.

1. Expansions of the second derivative of density.

$$
f^{\prime \prime}(x ; \alpha)=\frac{1}{\pi \alpha} \sum_{k=1}^{\infty} \frac{\Gamma((2 k+1) / \alpha)}{(2 k-2) !}(-1)^{k} x^{2 k-2},
$$

For $1<\alpha \leq 2$ this series is convergent for all $x$ and for $\alpha<1$ this is justified as an asymptotic expansion as $x \rightarrow 0$. Similarly for $x \rightarrow \infty$ we have

$$
f^{\prime \prime}(x ; \alpha)=\frac{1}{\pi} \sum_{k=1}^{\infty} \frac{\Gamma(\alpha k+3)}{k !}(-1)^{k-1} \sin \left(\frac{\pi \alpha k}{2}\right) x^{-k \alpha-3},
$$

For $\alpha<1$ this series is convergent for $x \neq 0$ and for $1<\alpha \leq 2$ this series is justified as an asymptotic expansion.

2. Taylor expansion of the second derivative of density around $\alpha=1$ :

$$
f^{\prime \prime}(x ; \alpha)=f^{\prime \prime}(x ; 1)+f_{\alpha}^{\prime \prime}(x ; 1)(\alpha-1)+\frac{1}{2} f_{\alpha \alpha}^{\prime \prime}(x ; 1)(\alpha-1)^{2}+o\left((\alpha-1)^{2}\right),
$$

where

$$
\begin{gathered}
f^{\prime \prime}(x ; 1)=\frac{6 x^{2}-2}{\pi\left(1+x^{2}\right)^{3}} \\
f_{\alpha}^{\prime \prime}(x ; 1)=\frac{2}{\pi}\left\{\frac{x^{4}-6 x^{2}+1}{\left(1+x^{2}\right)^{4}}\left(\frac{11}{2}-3 \gamma-\frac{3}{2} \log \left(1+x^{2}\right)\right)+\frac{12 x\left(x^{2}-1\right)}{\left(1+x^{2}\right)^{4}} \arctan x\right\},
\end{gathered}
$$


and

$$
\begin{aligned}
f_{\alpha \alpha}^{\prime \prime}(x ; 1)= & \frac{6\left(x^{6}-25 x^{4}+35 x^{3}-3\right)}{\pi\left(1+x^{2}\right)^{5}}\left\{\frac{\pi^{2}}{6}+\left(1-\gamma-\frac{1}{2} \log \left(1+x^{2}\right)\right)^{2}-1-\arctan ^{2} x\right\} \\
& +\frac{96 x\left(x^{4}-5 x^{2}+2\right)}{\pi\left(1+x^{2}\right)^{5}} \arctan x\left(\frac{3}{2}-\gamma-\frac{1}{2} \log \left(1+x^{2}\right)\right) \\
& +\frac{2\left(5 x^{6}-155 x^{4}+235 x^{2}-21\right)}{\pi\left(1+x^{2}\right)^{5}}\left(1-\gamma-\frac{1}{2} \log \left(1+x^{2}\right)\right) \\
& +\frac{4 x\left(11 x^{4}-70 x^{2}+31\right)}{\pi\left(1+x^{2}\right)^{5}} \arctan x+\frac{2\left(x^{6}-50 x^{4}+85 x^{2}-8\right)}{\pi\left(1+x^{2}\right)^{5}} .
\end{aligned}
$$

\begin{tabular}{|c|c|c|c|c|}
\hline$\alpha \backslash x$ & $x \rightarrow 0$, formu & $(4.6)$ & $x \rightarrow \infty$, formu & $(4.7)$ \\
\hline & number of terms $k$ & range of $x$ & number of terms $k$ & range of $x$ \\
\hline$[0.2,0.25]$ & \multirow{4}{*}{$k=5$} & $x<10^{-8}$ & \multirow{4}{*}{$k=10$} & \multirow{4}{*}{$x>10^{\frac{3}{1+\alpha}}$} \\
\hline$(0.25,0.3]$ & & $x<10^{-6}$ & & \\
\hline$(0.3,0.9]$ & & $x<10^{-5}$ & & \\
\hline$(0.9,0.99]$ & & $x<10^{-3}$ & & \\
\hline$(0.99,1.01]$ & \multicolumn{4}{|c|}{ formula (4.8) } \\
\hline$(1.01,1.02]$ & \multirow{3}{*}{$k=10$} & \multirow{3}{*}{$x<10^{-3}$} & & \\
\hline$(1.02,1.999]$ & & & $k=10$ & $x>10^{\frac{3}{1+\alpha}}$ \\
\hline$(1.999,2.0]$ & & & \multicolumn{2}{|c|}{ formula (4.5) } \\
\hline
\end{tabular}

Table 3: Approximations to $f^{\prime \prime}(x ; \alpha)$ at boundary cases

Table 3 summarizes uses of various expansions. Here formula (4.8) means use of Taylor expansion of the second derivative of density around $\alpha=1$ and (4.5) means direct use of integral expression of the second derivative. Note for $\alpha \rightarrow 2$ and $x \rightarrow \infty$ the same problem arise as in the density. However we do not use the second derivative of density for $\alpha \in(1.999,2.0]$ in this paper.

\section{Partial derivatives with respect to the characteristic exponent}

In this section we discuss the first and the second derivatives of the stable density with respect to the characteristic exponent $\alpha$.

\subsection{The first derivative w.r.t. $\alpha$}

We only need to investigate the standard ( $\mu=0$ and $\sigma=1)$ case. Although the representations of $f_{\alpha}(x ; \alpha)$ sometimes become complicated, careful analysis of various cases gives accurate and efficient calculations. The direct differentiation of (2.6) yields the following representation.

$$
f_{\alpha}(x ; \alpha)=\frac{1}{\alpha(1-\alpha)} f(x ; \alpha)+\frac{\alpha}{\pi|\alpha-1| x} \int_{0}^{\frac{\pi}{2}} g_{\alpha}(\varphi ; \alpha, x)(1-g(\varphi ; \alpha, x)) \exp (-g(\varphi ; \alpha, x)) d \varphi,
$$


where

$$
\begin{aligned}
g_{\alpha}(x ; \alpha)= & -\left(\frac{x \cos \varphi}{\sin \alpha \varphi}\right)^{\frac{\alpha}{\alpha-1}} \frac{\cos (\alpha-1)}{\cos \varphi} \\
& \times\left\{\frac{1}{(\alpha-1)^{2}} \log \left|\frac{x \cos \varphi}{\sin \alpha \varphi}\right|+\frac{\alpha \varphi}{\alpha-1} \frac{1}{\tan \alpha \varphi}+\varphi \tan (\alpha-1) \varphi\right\} \\
= & -g(x ; \alpha)\left\{\frac{1}{(\alpha-1)^{2}} \log \left|\frac{x \cos \varphi}{\sin \alpha \varphi}\right|+\frac{\alpha \varphi}{\alpha-1} \frac{1}{\tan \alpha \varphi}+\varphi \tan (\alpha-1) \varphi\right\} \\
= & -g(x ; \alpha)\left(h_{1}(\varphi)+h_{2}(\varphi)+h_{3}(\varphi)\right) .
\end{aligned}
$$

Here for convenience $h_{i}(\varphi), i=1,2,3$, are defined as

$$
h_{1}(\varphi)=\frac{1}{(\alpha-1)^{2}} \log \left|\frac{x \cos \varphi}{\sin \alpha \varphi}\right|, \quad h_{2}(\varphi)=\frac{\alpha \varphi}{\alpha-1} \frac{1}{\tan \alpha \varphi}, \quad h_{3}(\varphi)=\varphi \tan (\alpha-1) \varphi .
$$

We consider the integrand $g_{\alpha}(\cdot)(1-g(\cdot)) \exp (-g(\cdot))$ in (5.1) separately, dividing it into three parts $g(\cdot) h_{i}(\cdot)(1-g(\cdot)) \exp (-g(\cdot))$.

For each $g(\cdot) h_{i}(\cdot)(1-g(\cdot)) \exp (-g(\cdot)), i=1,2,3$, we divide the interval of integration appropriately based on zero points $\varphi_{i}, i=1,4,5$. The following properties are obtained by careful evaluation of the signs of derivatives of $h_{i}(\cdot)$.

$h_{1}(\varphi)$ : strictly decreasing, zero at $\varphi_{4}: \frac{x \cos \varphi}{\sin \alpha \varphi}=1, \varphi_{1}<\varphi_{4}$ for $\alpha<1$ and $\varphi_{4}<\varphi_{1}$ for $\alpha>1$.

$h_{2}(\varphi)$ : negative for $\alpha<1$. The sign changes from + to - at $\varphi_{5}=\frac{\pi}{2 \alpha}$ for $\alpha>1$.

$h_{3}(\varphi)$ : nonpositive for $\alpha<1$. nonnegative for $\alpha>1$.

As in the density, we use alternative representations of the first derivative of the symmetric stable density concerning $\alpha$ around the boundary and following representations are needed.

1. Expansions of the first derivative of the density w.r.t $\alpha$.

$$
f_{\alpha}(x ; \alpha)=-\frac{1}{\pi \alpha^{2}} \sum_{k=0}^{\infty} \frac{\Gamma^{\prime}((2 k+1) / \alpha+1)}{(2 k) !}\left(-x^{2}\right)^{k} .
$$

For $1<\alpha \leq 2$ this series is convergent for every $x$ and for $\alpha<1$ it is justified as an asymptotic expansion as $x \rightarrow 0$. Similarly for $x \rightarrow \infty$ we have

$$
\begin{aligned}
f_{\alpha}(x ; \alpha)= & \frac{1}{\pi} \sum_{k=1}^{\infty} \frac{\Gamma^{\prime}(\alpha k+1)}{(k-1) !}(-1)^{k-1} \sin \left(\frac{\pi \alpha k}{2}\right) x^{-k \alpha-1} \\
& +\frac{1}{\pi} \sum_{k=1}^{\infty} \frac{\Gamma(\alpha k+1)}{(k-1) !}(-1)^{k-1}\left[\frac{\pi}{2} \cos \left(\frac{\pi \alpha k}{2}\right)-\log x \sin \left(\frac{\pi \alpha k}{2}\right)\right] x^{-k \alpha-1}
\end{aligned}
$$

For $\alpha<1$ this series converges for every $x \neq 0$ and for $\alpha>1$ this series is justified as an asymptotic expansion.

2. Taylor expansion of the first derivative of $\alpha$ around $\alpha=1$ :

$$
f_{\alpha}(x ; \alpha)=f_{\alpha}(x ; 1)+f_{\alpha \alpha}(x ; 1)(\alpha-1)+o(|\alpha-1|),
$$

where $f_{\alpha}(x ; 1)$ and $f_{\alpha \alpha}(x ; 1)$ are given by $(3.2)$ and $(3.3)$. 
In order to first confirm the derivative at $\alpha=2$ we utilize equation 3.21 on page 12 of Oberhettinger (1990).

$$
\int_{0}^{\infty} u^{\nu} e^{-a u^{2}} \cos (u y) d u=\frac{1}{2}\left(\frac{\pi}{2}\right)^{\frac{1}{2}}(2 a)^{-\frac{1}{2}-\frac{1}{2} \nu} \sec \left(\frac{\pi \nu}{2}\right) \exp \left(-\frac{y^{2}}{8 a}\right)\left(D_{\nu}(z)+D_{\nu}(-z)\right),
$$

where $z=(2 a)^{-\frac{1}{2}} y$ and $D_{\nu}(z)$ is the parabolic cylinder functions (see p.255 of Oberhettinger (1990)). Differentiating this with respect to $\nu$, setting $\nu=2$ and adjusting some signs and constants we obtain $f_{\alpha}(x ; 2)$. These values coincide with that of expansions in (5.4) at $\alpha=2$. Note the integral (5.1) is not accurate when $\alpha$ is very close to 2 especially for $\alpha>1.9999999$. This inaccuracy of the integral (5.1) is illustrated in Figure 6, in comparison to the accurate values shown in Figure 5.

Table 4 is a summary of approximations to the first derivative of the density function with respect to $\alpha$.

The formula (5.6) means Taylor expansion of the first derivative of $\alpha$ around $\alpha=1$. For $\alpha \in$ $(1.9999,2.0]$ we use only the expansion (5.4) and the asymptotic expansion (5.5).

Table 4: Approximations to $f_{\alpha}(x ; \alpha)$ at boundary cases

\begin{tabular}{|c|c|c|c|c|}
\hline$\alpha \backslash x$ & \multicolumn{2}{|c|}{$x \rightarrow 0$, formula $(5.4)$} & \multicolumn{2}{|c|}{$x \rightarrow \infty$, formula $(5.5)$} \\
\hline & number of terms $k$ & range of $x$ & number of terms $k$ & range of $x$ \\
\hline$[0.1,0.2]$ & $k=1$ & $x<10^{-16}$ & \multirow{4}{*}{$k=10$} & \multirow{4}{*}{$x>10^{\frac{3}{1+\alpha}}$} \\
\hline$(0.2,0.3]$ & \multirow{3}{*}{$k=5$} & $x<10^{-7}$ & & \\
\hline$(0.3,0.5]$ & & $x<10^{-5}$ & & \\
\hline$(0.5,0.99]$ & & $x<10^{-5}$ & & \\
\hline$(0.99,1.01]$ & \multicolumn{4}{|c|}{ formula (5.6) } \\
\hline$(1.01,1.9999]$ & $k=10$ & $x<10^{-5}$ & $k=10$ & $x>10^{\frac{3}{1+\alpha}}$ \\
\hline$(1.9999,2.0]$ & $k=85$ & $x \leq 8.0$ & $k=20$ & $x>8.0$ \\
\hline
\end{tabular}

Here we present graphs of the first derivative w.r.t. $\alpha$ in Figure 3, Figure 4, Figure 5, Figure 6, Figure 7 and Figure 8. The first derivative $f_{\alpha}(x ; \alpha)$ with respect to $\alpha$ is shown in Figure 3 and 4 and the score function $f_{\alpha}(x ; \alpha) / f(x ; \alpha)$ is shown in Figure 7 and 8 . Note that graphs of $f_{\alpha}(x ; \alpha)$ where $\alpha=1.999999$ and $\alpha=2.0$ in Figure 5 are almost the same corresponding to the fact $f_{\alpha}(x ; \alpha)$ is continuous in $\alpha \in(0,2]$.

\subsection{The second derivative w.r.t. $\alpha$}

Differentiating (5.1) we obtain

$$
\begin{aligned}
& f_{\alpha \alpha}(x ; \alpha)=\frac{2}{\alpha(1-\alpha)^{2}} f(x ; \alpha) \\
& \quad+\frac{2 \operatorname{sgn}(\alpha-1)}{\pi(\alpha-1)^{2} x} \int_{0}^{\frac{\pi}{2}} g(\varphi)\left\{(1+\alpha) h_{1}(\varphi)+2 h_{2}(\varphi)+h_{3}(\varphi)\right\}(1-g(\varphi)) \exp (-g(\varphi)) d \varphi \\
& \quad+\frac{\alpha}{\pi|\alpha-1| x} \int_{0}^{\frac{\pi}{2}} g(\varphi)\left(g^{2}(\varphi)-3 g(\varphi)+1\right)\left\{h_{1}(\varphi)+h_{2}(\varphi)+h_{3}(\varphi)\right\}^{2} \exp (-g(\varphi)) d \varphi \\
& \quad+\frac{\alpha}{\pi|\alpha-1| x} \int_{0}^{\frac{\pi}{2}} g(\varphi)\left(\frac{\alpha}{\alpha-1} \frac{\varphi^{2}}{\sin ^{2} \alpha \varphi}-\frac{\varphi^{2}}{\cos ^{2}(\alpha-1) \varphi}\right)(1-g(\varphi)) \exp (-g(\varphi)) d \varphi .
\end{aligned}
$$




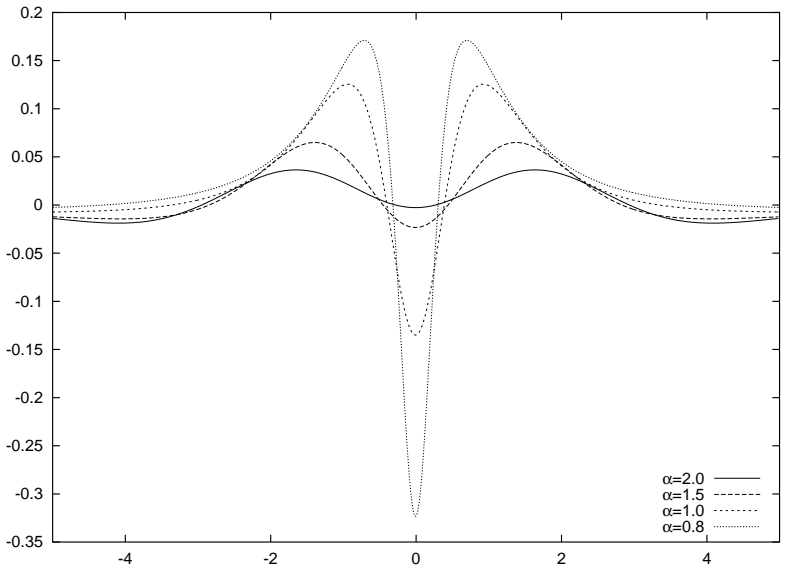

Figure 3: First derivative $f_{\alpha}(x ; \alpha)$ w.r.t. to $\alpha$

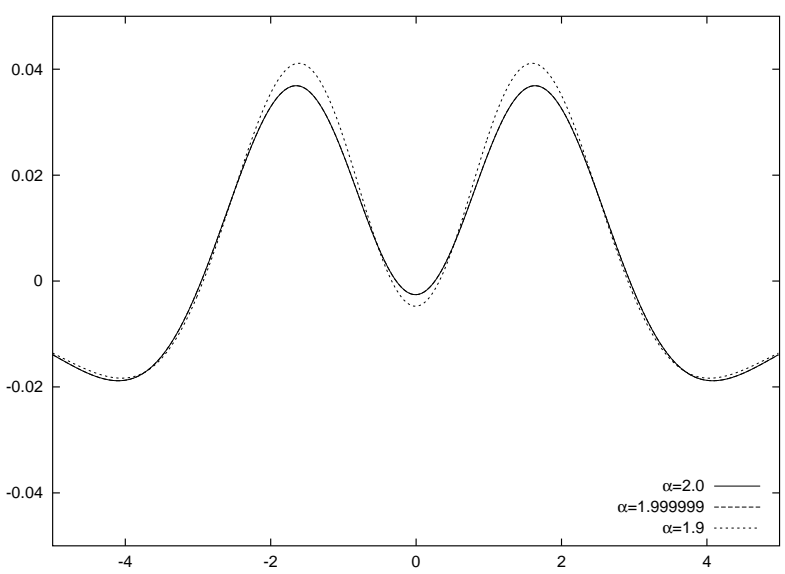

Figure 5: $f_{\alpha}(x ; \alpha)$ (accurate)

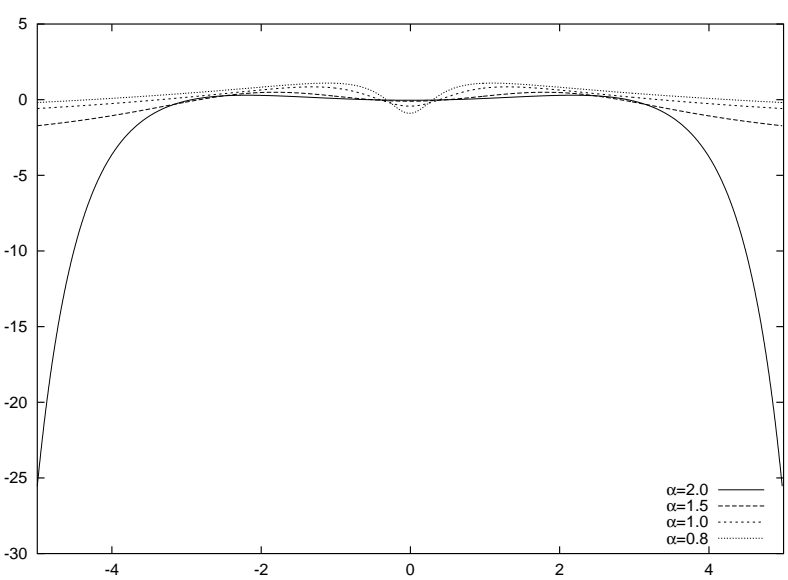

Figure 7: Score function $f_{\alpha}(x ; \alpha) / f(x ; \alpha)$

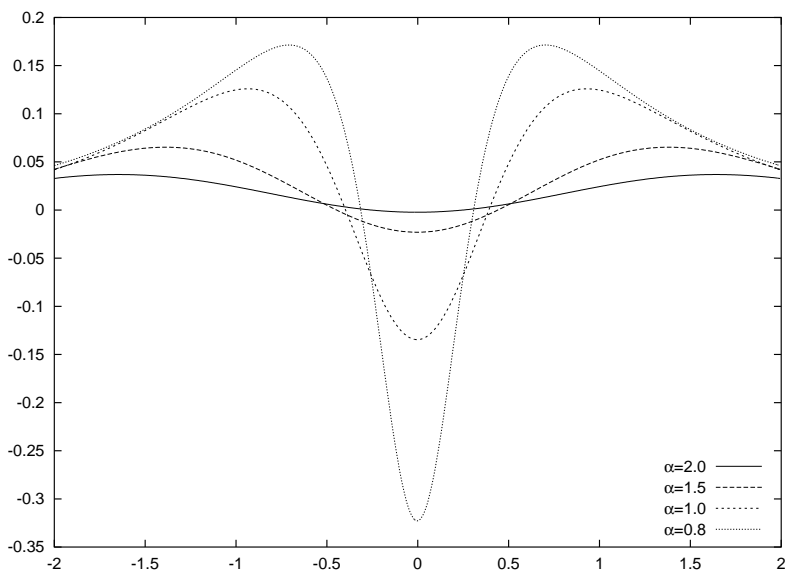

Figure 4: $f_{\alpha}(x ; \alpha)$ (more detail around 0)

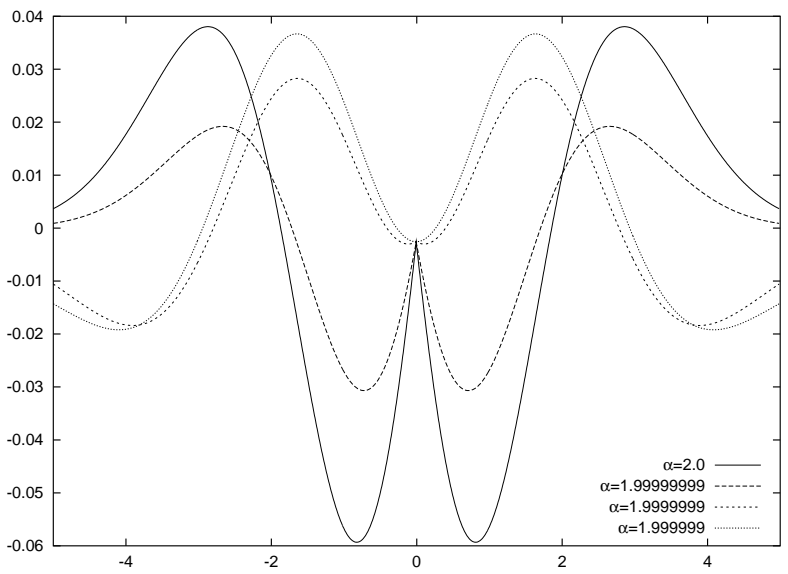

Figure 6: $f_{\alpha}(x ; \alpha)$ using (5.1) (not accurate)

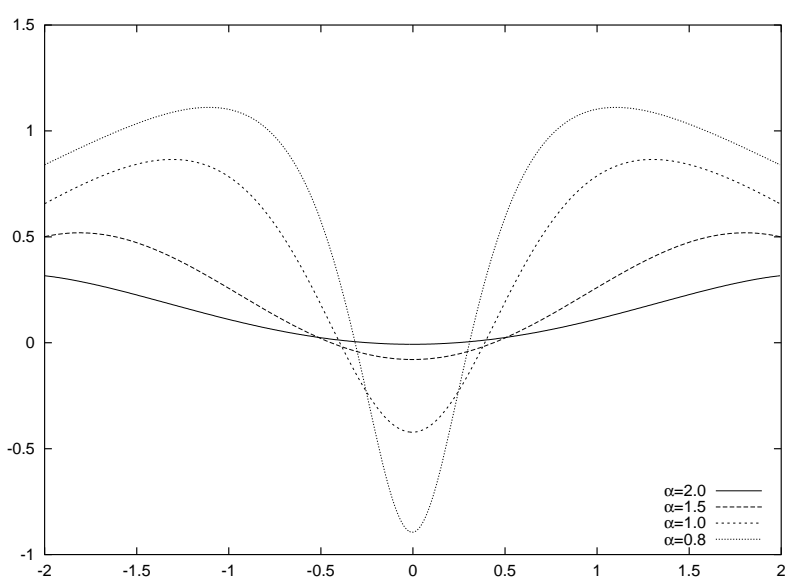

Figure 8: $f_{\alpha}(x ; \alpha) / f(x ; \alpha)$ (more detail around 0 ) 
Concerning the second integral we utilize the arguments of Section 4.1 for efficient integration. The third integral is calculated by dividing the interval of integration using zeros of integrand $\varphi_{i}$ for $i=1,2,3$. As for the fourth integral, we integrate separately the two terms in the integrand. An alternative representations of the second derivative of the symmetric stable density concerning $\alpha$ around the boundary are as follows.

1. Expansions of the second derivative of density w.r.t. $\alpha$.

$$
\begin{aligned}
f_{\alpha \alpha}(x ; \alpha)= & \frac{2}{\pi \alpha^{3}} \sum_{k=0}^{\infty} \frac{\Gamma^{\prime}((2 k+1) / \alpha+1)}{(2 k) !}\left(-x^{2}\right)^{k} \\
& +\frac{1}{\pi \alpha^{4}} \sum_{k=0}^{\infty} \frac{\Gamma^{\prime \prime}((2 k+1) / \alpha+1)}{(2 k) !}(2 k+1)\left(-x^{2}\right)^{k} .
\end{aligned}
$$

For $1<\alpha \leq 2$ this series is convergent for all $x$ and for $\alpha<1$ this is justified as an asymptotic expansion as $x \rightarrow 0$. Similarly for $x \rightarrow \infty$ we have

$$
\text { (5.9) } \begin{aligned}
f_{\alpha \alpha}(x ; \alpha)= & \frac{1}{\pi} \sum_{k=1}^{\infty} \frac{\Gamma^{\prime \prime}(\alpha k+1)}{(k-1) !} k(-1)^{k-1} \sin \left(\frac{\pi \alpha k}{2}\right) x^{-k \alpha-1} \\
& -\frac{2}{\pi} \sum_{k=1}^{\infty} \frac{\Gamma^{\prime}(\alpha k+1)}{(k-1) !} k(-1)^{k}\left[\frac{\pi}{2} \cos \left(\frac{\pi \alpha k}{2}\right)-\log x \sin \left(\frac{\pi \alpha k}{2}\right)\right] x^{-k \alpha-1} \\
& +\frac{1}{\pi} \sum_{k=1}^{\infty} \frac{\Gamma(\alpha k+1)}{(k-1) !} k(-1)^{k-1}\left[\left(\log ^{2}(x)-\frac{\pi^{2}}{4}\right) \sin \left(\frac{\pi \alpha k}{2}\right)\right. \\
& \left.-\pi \log x \cos \left(\frac{\pi \alpha k}{2}\right)\right] x^{-k \alpha-1} .
\end{aligned}
$$

For $\alpha<1$ this series is convergent for $x \neq 0$ and for $1<\alpha \leq 2$ this series is justified as an asymptotic expansion.

2. Taylor expansion of the second derivative of $\alpha$ around $\alpha=1$ :

$$
f_{\alpha \alpha}(x ; \alpha)=f_{\alpha \alpha}(x ; 1)+f_{\alpha \alpha \alpha}(x ; 1)(\alpha-1)+o(|\alpha-1|),
$$

where $f_{\alpha \alpha \alpha}(x ; 1)$ is given in Appendix $\mathrm{A}$.

Table 5 is a summary of approximations of the second derivative of density function concerning $\alpha$. Formula (5.10) means use of Taylor expansion of the second derivative of $\alpha$ around $\alpha=1$. For the sake of convenience we use the integral representation (5.7) when $\alpha \in(1.999,2.0)$ and $x \rightarrow \infty$. This is somewhat problematic as in the case of the first derivative w.r.t. $\alpha$ and further investigation is needed for $\alpha$ near 2. In the calculations of the present paper, this problem appears only in the observed fisher information for $\alpha=1.5$. However the estimated values $\hat{\alpha}$ very near $\alpha=2$ seldom occur and there seems to be no influence of this problem in the values presented in Table 8.

\section{$6 \quad$ Fisher informations of symmetric stable distributions}

Using the results of the previous section we can accurately evaluate the Fisher information matrices of the symmetric stable distributions. To the authors' knowledge there are only two somewhat 
Table 5: Approximations to $f_{\alpha \alpha}(x ; \alpha)$ at boundary cases

\begin{tabular}{|c|c|c|c|c|}
\hline$\alpha \backslash x$ & \multicolumn{2}{|c|}{$x \rightarrow 0$, formula $(5.8)$} & \multicolumn{2}{|c|}{$x \rightarrow \infty$, formula $(5.9)$} \\
\hline & number of terms $k$ & range of $x$ & number of terms $k$ & range of $x$ \\
\hline$[0.2,0.3]$ & \multirow{3}{*}{$k=5$} & $x<2.0 \times 10^{-7}$ & \multirow{3}{*}{$k=10$} & \multirow{3}{*}{$x>10^{\frac{3}{1+\alpha}}$} \\
\hline$(0.3,0.5]$ & & $x<10^{-5}$ & & \\
\hline$(0.5,0.99]$ & & $x<10^{-3}$ & & \\
\hline$(0.99,1.03]$ & \multicolumn{4}{|c|}{ formula (5.10) } \\
\hline$(1.01,1.999]$ & \multirow{2}{*}{$k=10$} & \multirow{2}{*}{$x<10^{-3}$} & $k=10$ & $x>10^{\frac{3}{1+\alpha}}$ \\
\hline$(1.999,2.0)$ & & & \multicolumn{2}{|c|}{ formula (5.7) } \\
\hline
\end{tabular}

incomplete results concerning the Fisher Informations of stable distributions. One is Nolan (2001) and the other is DuMouchel (1975). The former was based on numerical differentiations of densities and did not give the informations for $\alpha<0.5$. The latter had the problem of truncation of the integral and did not give the informations for $\alpha<0.8$. Considering that the tails and modes of distributions are important for calculating informations, our approach is desirable. We give the informations $\alpha \in[0.2,2.0]$ of the symmetric distributions in Table 6 . In Table 6 ' $*$ ' means that the quantity is not defined. The values in Table 6 largely coincide with those of DuMouchel (1975).

The components of Fisher information matrix $I$ are defined as follows.

$$
I_{i j}=\int_{-\infty}^{\infty} \frac{\partial f}{\partial \theta_{i}} \frac{\partial f}{\partial \theta_{j}} \frac{1}{f} d x
$$

where $\left(\theta_{1}, \theta_{2}, \theta_{3}\right)=(\mu, \sigma, \alpha)$. By symmetry it can be easily shown that $I_{12}=I_{21}=0$. For notational clarity we write $I_{11}=I_{\mu \mu}, I_{22}=I_{\sigma \sigma}, I_{23}=I_{\sigma \alpha}$ and $I_{33}=I_{\alpha \alpha}$. For numerical calculations of (6.1) we use adaptive integration of infinite intervals with singularities (QUAGIU) in GNU Scientific Library (2003). In can be shown that the information matrix at the Cauchy $(\alpha=1.0)$ is analytically given as

$$
I_{\mu \mu}=I_{\sigma \sigma}=1 / 2 \sigma^{2}, \quad I_{\sigma \alpha}=\frac{1}{2 \sigma}(1-\gamma-\log 2), \quad I_{\alpha \alpha}=\frac{1}{2}\left\{\frac{\pi^{2}}{6}+(\gamma+\log 2-1)^{2}\right\} .
$$

These values are consistent with numerical calculations in Table 6 .

In order to check our computations, we have done small simulation study of maximum likelihood estimation of $\alpha$ (for fixed $\mu=0$ and $\sigma=1$ ). We found that simulated information coincides well with the exact information. Table 7 is the results of 1000 iterations of maximum likelihood estimation of $\alpha$ with the sample size of $n=50$. We denote the maximum likelihood estimator by $\hat{\alpha} . \bar{\alpha}$ is the average of 1000 iterations and $\hat{\sigma}_{\alpha}^{2}$ is the variance of $\sqrt{50} \times(\hat{\alpha}-\alpha)$. Though the convergence of $1 / \hat{\sigma}_{\alpha}^{2}$ to $I_{\alpha \alpha}$ seems somewhat slow for $\alpha<1$, the values of $1 / \hat{\sigma}_{\alpha}^{2}$ are consistent with the exact Fisher information $I_{\alpha \alpha}$.

Furthermore we simulated the observed Fisher information (2.5) of $\alpha$ in Table 8. Here $\hat{I}_{\alpha \alpha}(2)$ corresponds to the right hand side of (2.5) and

$$
\hat{I}_{\alpha \alpha}(1)=\frac{1}{n} \sum_{i=1}^{n}\left(\frac{f_{\alpha}\left(x_{i} ; \hat{\alpha}\right)}{f\left(x_{i} ; \hat{\alpha}\right)}\right)^{2}
$$

involves the first derivative only. Variance of $\hat{I}_{\alpha \alpha}(1)$ and $\hat{I}_{\alpha \alpha}(2)$ are also shown in the parentheses in Table 8. $\hat{I}_{\alpha \alpha}(2)$ has smaller variance than $\hat{I}_{\alpha \alpha}(1)$ but some positive bias is observed in $\hat{I}_{\alpha \alpha}(2)$ compared to the true information $I_{\alpha \alpha}$. 
Table 6: Fisher information matrix of symmetric stable distributions

\begin{tabular}{ccccc}
\hline$\alpha \backslash I_{i j}$ & $I_{\mu \mu}$ & $I_{\sigma \sigma}$ & $I_{\alpha \alpha}$ & $I_{\sigma \alpha}$ \\
\hline 2.0 & 0.5 & 2.0 & $\infty$ & $*$ \\
1.999 & 0.4995 & 1.9904 & 29.461 & -0.8685 \\
1.99 & 0.4960 & 1.9321 & 4.6197 & -0.6682 \\
1.95 & 0.4842 & 1.7631 & 1.4108 & -0.4821 \\
1.9 & 0.4727 & 1.6127 & 0.8846 & -0.3963 \\
1.8 & 0.4552 & 1.3898 & 0.5937 & -0.3138 \\
1.7 & 0.4424 & 1.2189 & 0.5028 & -0.2692 \\
1.6 & 0.4334 & 1.0775 & 0.4726 & -0.2396 \\
1.5 & 0.4281 & 0.9556 & 0.4737 & -0.2174 \\
1.4 & 0.4270 & 0.8475 & 0.4973 & -0.1992 \\
1.3 & 0.4310 & 0.7498 & 0.5424 & -0.1832 \\
1.2 & 0.4419 & 0.6603 & 0.6119 & -0.1679 \\
1.1 & 0.4630 & 0.5774 & 0.7132 & -0.1523 \\
1.05 & 0.4790 & 0.5381 & 0.7794 & -0.1440 \\
1.01 & 0.4953 & 0.5075 & 0.8413 & -0.1369 \\
1.0 & 0.5 & 0.5 & 0.8590 & -0.1352 \\
0.99 & 0.5049 & 0.4925 & 0.8763 & -0.1332 \\
0.95 & 0.5276 & 0.4631 & 0.9552 & -0.1257 \\
0.9 & 0.5641 & 0.4272 & 1.0721 & -0.1154 \\
0.8 & 0.6800 & 0.3586 & 1.3928 & -0.0913 \\
0.7 & 0.9094 & 0.2937 & 1.8974 & -0.0611 \\
0.6 & 1.4446 & 0.2325 & 2.7414 & -0.0220 \\
0.5 & 3.1167 & 0.1753 & 4.2748 & 0.0295 \\
0.4 & 12.256 & 0.1226 & 7.3994 & 0.0979 \\
0.3 & 188.09 & 0.0756 & 14.925 & 0.1869 \\
0.2 & 149359.4 & 0.0367 & 38.729 & 0.2938
\end{tabular}

Table 7: MLE $(n=50,1000$ iteration $)$

\begin{tabular}{cccc}
\hline$\alpha$ & $\bar{\alpha}$ & $1 / \hat{\sigma}_{\alpha}$ & $I_{\alpha \alpha}$ \\
\hline 1.99 & 1.974 & 4.4877 & 4.6197 \\
1.8 & 1.811 & 0.6396 & 0.5937 \\
1.7 & 1.714 & 0.5102 & 0.5028 \\
1.5 & 1.531 & 0.4532 & 0.4737 \\
1.3 & 1.320 & 0.5114 & 0.5424 \\
1.0 & 1.027 & 0.7676 & 0.8590 \\
0.8 & 0.819 & 1.1944 & 1.3928 \\
0.5 & 0.509 & 3.8803 & 4.2748
\end{tabular}


Table 8: Observed information (variance) $(n=50,1000$ iteration)

\begin{tabular}{ccccccc}
\hline$\alpha$ & $\bar{\alpha}$ & \multicolumn{2}{c}{$\hat{I}_{\alpha \alpha}(1)$} & \multicolumn{2}{c}{$\hat{I}_{\alpha \alpha}(2)$} & $I_{\alpha \alpha}$ \\
\hline 1.5 & 1.531 & 0.4863 & $(0.033)$ & 0.5145 & $(0.018)$ & 0.4737 \\
1.0 & 1.025 & 0.8541 & $(0.157)$ & 0.9001 & $(0.099)$ & 0.8590 \\
0.5 & 0.509 & 4.2819 & $(3.517)$ & 4.4660 & $(2.755)$ & 4.2748 \\
\hline
\end{tabular}

For the rest of this section, we discuss behavior of the Fisher information as $\alpha \uparrow 2$. Investigation of the Fisher information in this case is difficult because it involves detailed study of behavior of the score function as $\alpha \uparrow 2$ and $x \rightarrow \infty$.

DuMouchel $(1975,1983)$ have proved that $I_{\alpha \alpha} \rightarrow \infty$ as $\alpha \uparrow 2$. Nagaev and Shkol'nik (1988) made more detailed analysis of $I_{\alpha \alpha}$ and shown that

$$
I_{\alpha \alpha}=\frac{1}{4 \Delta \log (1 / \Delta)}(1+o(1)), \quad \Delta=2-\alpha .
$$

Table 9 gives the information for $\alpha$ extremely close to 2. $I_{\alpha \alpha}(1)$ means use of Taylor approximation around normal (3.5) and $I_{\alpha \alpha}(2)$ means use of $(2.9)$ when $x>10^{\frac{3}{1+\alpha}}$ for the density. The same is done with $I_{\sigma \alpha}$. There seems to be no substantial difference between $I_{\alpha \alpha}(1)$ and $I_{\alpha \alpha}(2)$. In the column N\&S we show the values of $1 /(4 \Delta \log (1 / \Delta))$. Our computation for $\Delta \geq 10^{-6}$ is accurate. The convergence of (6.3) seems to be very slow. Limiting theoretical behavior of $I_{\sigma \alpha}(\alpha)$ as $\alpha \uparrow 2$ is not known at present.

Table 9: Information around $\alpha=2$

\begin{tabular}{llllll}
\hline$\alpha$ & $I_{\alpha \alpha}(1)$ & $I_{\alpha \alpha}(2)$ & $\mathrm{N} \& \mathrm{~S}$ & $I_{\sigma \alpha}(1)$ & $I_{\sigma \alpha}(2)$ \\
\hline $2.0-10^{-10}$ & 106860414 & 92384764 & 108573620 & -1.3482 & -1.3427 \\
$2.0-10^{-9}$ & 10810787 & 10167389 & 12063736 & -1.3482 & -1.3395 \\
$2.0-10^{-8}$ & 1144778 & 1131645 & 1357170 & -1.3482 & -1.3123 \\
$2.0-10^{-7}$ & 127953 & 127802 & 155105.2 & -1.3478 & -1.2553 \\
$2.0-10^{-6}$ & 14724 & 14722 & 18095.60 & -1.2094 & -1.1923 \\
1.99999 & 1750 & 1750 & 2171.472 & -1.1100 & -1.1100 \\
1.9999 & 217 & 217 & 271.4341 & -1.0069 & -1.0069
\end{tabular}

\section{Conclusions and some discussions}

In this paper we proposed reliable numerical calculations of the symmetric stable densities and their partial derivatives including various boundary cases. We found that except for very small values of $\alpha$ $(\alpha<0.1)$ our method works very well. This enables us to reliably compute the maximum likelihood estimator of the symmetric stable distributions and its standard error.

For the family of stable distributions, the use of the observed Fisher information in (2.5) for assessing the standard deviation of the maximum likelihood estimator needs further investigation. 
Our simulation suggests that there may be some merit in using only the first term on the right hand side of (2.5).

Further study is needed to theoretically establish the limiting behavior of the Fisher information matrix as $\alpha \uparrow 2$.

Finally it is of interest to extend the methods of the present paper to the general asymmetric stable densities and to the multivariate symmetric stable densities. These extensions will be studied in our subsequent works.

\section{A The third order derivative of $f(x ; \alpha)$ w.r.t. $\alpha$ around Cauchy}

Write

$$
G(\nu, y)=\int_{0}^{\infty} u^{\nu-1} e^{-u} \log ^{3} u \cos (u y) d u
$$

Differentiating (3.4) three times and setting $a=1$ we get

$$
\begin{gathered}
G(\nu, y)=\left(1+y^{2}\right)^{-\frac{1}{2} \nu}\left\{\frac{\Gamma(\nu)}{4} \log ^{2}\left(1+y^{2}\right)-\Gamma^{\prime}(\nu) \log \left(1+y^{2}\right)+\Gamma^{\prime \prime}(\nu)\right\} \\
\quad \times\left\{\cos (\nu z)\left(\psi(\nu)-\frac{1}{2} \log \left(1+y^{2}\right)\right)-z \sin (\nu)\right\} \\
+\left(1+y^{2}\right)^{-\frac{1}{2} \nu}\left\{-\Gamma(\nu) \log \left(1+y^{2}\right)+2 \Gamma^{\prime}(\nu)\right\} \\
\quad \times\left\{-z \sin (\nu z)\left(\psi(\nu)-\frac{1}{2} \log \left(1+y^{2}\right)\right)-z^{2} \cos (\nu z)+\cos (\nu z) \psi^{\prime}(\nu z)\right\} \\
+\left(1+y^{2}\right)^{-\frac{1}{2} \nu} \Gamma(\nu)\left[-z^{2} \cos (\nu z)\left(\psi(\nu)-\frac{1}{2} \log \left(1+y^{2}\right)\right)\right. \\
\left.-2 z \sin (\nu z) \psi^{\prime}(\nu)+z^{3} \sin (\nu z)+\cos (\nu z) \psi^{\prime \prime}(\nu)\right],
\end{gathered}
$$

where $z=\arctan y$. Then

$$
f_{\alpha \alpha \alpha}(x ; 1)=\frac{1}{\pi}(-G(4, x)+3 G(3, x)-G(2, x)) .
$$

\section{B Derivatives of the density at $\alpha=0.5$}

For the purpose of checking some of our calculations, we can use the explicit formula of the density at the special case of $\alpha=0.5$. From (2.8.30) of Zolotarev (1986) $f(x ; 0.5)$ is written as

$$
f(x ; 0.5)=\frac{x^{-\frac{3}{2}}}{\sqrt{2 \pi}}\left[\sin \left(\frac{1}{4 x}\right)\left\{\frac{1}{2}-S\left(\frac{1}{\sqrt{2 \pi x}}\right)\right\}+\cos \left(\frac{1}{4 x}\right)\left\{\frac{1}{2}-C\left(\frac{1}{\sqrt{2 \pi x}}\right)\right\}\right]
$$

where

$$
S(x)=\int_{0}^{x} \sin \left(\frac{\pi}{2} t^{2}\right) \quad \text { and } \quad C(x)=\int_{0}^{x} \cos \left(\frac{\pi}{2} t^{2}\right)
$$


are known as Fresnel integral functions. We differentiate the above representation and obtain

$$
\begin{aligned}
f^{\prime}(x ; 0.5)= & -\frac{3}{2} \frac{x^{-\frac{5}{2}}}{\sqrt{2 \pi}}\left[\sin \left(\frac{1}{4 x}\right)\left\{\frac{1}{2}-S\left(\frac{1}{\sqrt{2 \pi x}}\right)\right\}+\cos \left(\frac{1}{4 x}\right)\left\{\frac{1}{2}-C\left(\frac{1}{\sqrt{2 \pi x}}\right)\right\}\right] \\
& -\frac{x^{-\frac{7}{2}}}{4 \sqrt{2 \pi}}\left[\cos \left(\frac{1}{4 x}\right)\left\{\frac{1}{2}-S\left(\frac{1}{\sqrt{2 \pi x}}\right)\right\}-\sin \left(\frac{1}{4 x}\right)\left\{\frac{1}{2}-C\left(\frac{1}{\sqrt{2 \pi x}}\right)\right\}\right]+\frac{x^{-3}}{4 \pi}
\end{aligned}
$$

and

$$
\begin{aligned}
f^{\prime \prime}(x ; 0.5)= & \frac{x^{-\frac{7}{2}}}{4 \sqrt{2 \pi}}\left(15-\frac{x^{-2}}{4}\right)\left[\sin \left(\frac{1}{4 x}\right)\left\{\frac{1}{2}-S\left(\frac{1}{\sqrt{2 \pi x}}\right)\right\}+\cos \left(\frac{1}{4 x}\right)\left\{\frac{1}{2}-C\left(\frac{1}{\sqrt{2 \pi x}}\right)\right\}\right] \\
& +\frac{5}{4} \frac{x^{-\frac{9}{2}}}{\sqrt{2 \pi}}\left[\cos \left(\frac{1}{4 x}\right)\left\{\frac{1}{2}-S\left(\frac{1}{\sqrt{2 \pi x}}\right)\right\}-\sin \left(\frac{1}{4 x}\right)\left\{\frac{1}{2}-C\left(\frac{1}{\sqrt{2 \pi x}}\right)\right\}\right]-\frac{9}{8} \frac{x^{-4}}{\pi} .
\end{aligned}
$$

We have confirmed that our formulas in Section 4 numerically coincide with these explicit expressions at $\alpha=1 / 2$ including the boundary cases.

\section{References}

[1] Bergström, H. (1953). On some expansions of stable distribution functions. Ark. Mat., 2, 375-378.

[2] Brorsen, B. W. and Yang, S. R. (1990). Maximum likelihood estimates of symmetric stable distribution parameters. Comm. Statist. Simulation Comput., 19, 1459-1464.

[3] DuMouchel, W. H. (1973). On the asymptotic normality of the maximum-likelihood estimate when sampling from a stable distribution. Ann. Statist., 1, 948-957.

[4] DuMouchel, W. H. (1975). Stable distributions in statistical inference 2: Information from stably distributed samples. J. Amer. Statist. Assoc., 70, 386-393.

[5] DuMouchel, W. H. (1983). Estimating the stable index $\alpha$ in order to measure tail thickness: a critique. Ann. Statist., 11, 1019-1031.

[6] Efron, B. and Hinkley, D. V. (1978). Assessing the accuracy of the maximum likelihood estimator: Observed versus expected Fisher information. Biometrika, 65, 457-481.

[7] Feller, W. (1971). An Introduction to Probability Theory and Its Applications. Vol. 2, 2nd ed., Wiley, New York.

[8] Free Software Foundation, Inc. (2003). GNU Scientific Library - Reference Manual. Edition 1.4, for GSL Version 1.4, 59 Temple Place - Suite 330, Boston, MA 02111, USA.

[9] McCulloch, J. H. (1998). Numerical approximation of the symmetric stable distribution and density. A Practical Guide to Heavy Tails (R. J. Adler et al. eds.), Birkhauser, Boston, 489-499.

[10] Nagaev, A. V. and Shkol'nik, S. M. (1988). Some properties of symmetric stable distributions close to the normal distribution. Theory of Probability and its Applications, 33, 139-144.

[11] Nolan, J. P. (1997). Numerical calculation of stable densities and distribution. Comm. Statist. Stochastic Models, 13, 759-774. 
[12] Nolan, J. P. (1998) Parameterizations and modes of stable distributions. Statist. Prob. Lett., 38, 187-195.

[13] Nolan, J. P. (2001). Maximum likelihood estimation and diagnostics for stable distributions. Lévy Processes: Theory and Applications (O. E. Barndorff-Nielsen et al. eds.), Birkhauser, Boston, 379-400.

[14] Oberhettinger, F. (1990). Tables of Fourier Transforms and Fourier Transforms of Distributions. Springer-Verlag, Berlin.

[15] Zolotarev, V. M. (1986). One-Dimensional Stable Distributions. Transl. of Math. Monographs, 65, Amer. Math. Soc., Providence, RI. (Transl. of the original 1983 Russian) 